\title{
Hydrodynamic Model for Particle Size Segregation in Granular Media
}

\author{
Leonardo Trujillo ${ }^{1}$ and Hans J. Herrmann ${ }^{1,2}$ \\ ${ }^{1}$ Laboratoire de Physique et Mécanique des Milieux Hétérogènes, UMR-CNRS \\ 7636, École Supérieure de Physique et de Chimie Industrielles, 10 rue Vauquelin, \\ 75231 Paris Cedex 05, France \\ ${ }^{2}$ Institut für Computeranwendungen 1, Universität Stuttgart, Pfaffenwaldring, 27, \\ D-70569 Stuttgart, Germany
}

\begin{abstract}
We present a hydrodynamic theoretical model for "Brazil nut" size segregation in granular materials. We give analytical solutions for the rise velocity of a large intruder particle immersed in a medium of monodisperse fluidized small particles. We propose a new mechanism for this particle size-segregation due to buoyant forces caused by density variations which come from differences in the local "granular temperature". The mobility of the particles is modified by the energy dissipation due to inelastic collisions and this leads to a different behavior from what one would expect for an elastic system. Using our model we can explain the size ratio dependence of the upward velocity.
\end{abstract}

Key words: granular materials, Brazil-nut effect, segregation, buoyancy PACS: 45.70.Mg, 05.20.Dd, 05.70.Ln

\section{Introduction}

The physics of granular materials is a subject of current interest [1]. A granular medium is a system of many macroscopic heterogeneous particles with dissipative interactions. One of the outstanding problems is the so-called "Brazil Nut effect" [2]: When a large intruder particle placed at the bottom of a vibrated bed tends to the top. This size segregation is due to the nonequilibrium, dissipative nature of granular media. Granular materials are handled in many industries. Many industrial machines that transport granular materials use vertical vibration to fluidize the material, and the quality of many products is affected by segregation. Size segregation is one of the most intriguing phenomena found in granular physics. A deeper understanding of this effect is 
therefore interesting for practical applications, and also represent theoretical challenge.

A series of experiments [3-12] and computer simulations [2,13-23] have elucidated different size segregation mechanisms, including vibration frequency and amplitude [3-8,14,19]; particle size [2,3,5,6,13-15,17,18,23] and size distribution $[16,19]$; particle shape [21]; and other properties such as density [9-12,17,22,23] and elastic modulus [19].

Several possible mechanisms for size segregation have been proposed. One is segregation in presence of convection observed experimentally in three dimensions by Knight et al., [4], and by Duran et al., in two dimensions [5], under conditions of low amplitude and high acceleration vibration. In this case, both intruder and the small particles are driven up along the middle of the cell, and while the smaller particles are carried down in a convection roll near the walls the intruder remains trapped on the top. In experiments performed by Vanel et al., in three dimensions, they observed two convective regimes separated by a critical frequency [7]. The first regime is associated with heaping and the second regime is similar to the one observed in Ref. [4]. Also, they reported a nonconvective regime observing a size dependent rise velocity. Employing large molecular dynamics simulations in two dimensions Pöschel and Herrmann [16], and in three dimension Gallas et al., [19], have recovered several aspects that are seen in experiments and recognize the lack of a theoretical description of the exact mechanism driving the segregation and the role of convection.

Other segregation mechanism is associated to the percolation of small grains. Based on a Monte Carlo computer simulation, Rosato et al., [2,13] argue that each cycle of the applied vibration causes all the grains to detach from the base of the container. Then, the smaller particles fall relatively freely, while the larger particles require larger voids to fall downward. The large grain therefore effectively rise through the bed. In the context of large-amplitude, low-frequency vertical shaking process (tapping), Jullien et al., predict a critical size ratio below which segregation does not occur $[14,15]$. This provoked some controversy [24-27] and this threshold may be an artifact of the simulation model based on the "steepest descent algorithm" [14]. Experiments in Helle Shaw cells $[3,5,6]$ observed an intruder size dependent behavior, where the segregation rate increases with the size ratio between the intruder and the surrounding particles. Duran et al., formulated a geometrical theory for segregation based on the arching effect [3]. They also claim experimental evidence for a segregation size threshold [5]. In this picture the intruder contributes to the formation of an arch sustained on small grains on both sides. Between each agitation the small particles tend to fill the region below the arch. So, at each cycle the small particles move downward and the intruder effectively rises. Using a modification of the algorithm proposed by Rosato et al., Dippel and Luding find a good qualitative agreement with the non-convective and 
size-dependent rising [18].

In another context, Caglioti et al., considered the geometrical properties of mixtures in the presence of compaction [21]. They established a relation for the effective mobilities of different particles in heterogeneous situations.

The effect of the intruder density was studied experimentally by Shinbrot and Muzzio [9], and Möbius et al., [11] in three dimensions, and by Liffman et al., [10] in two dimensions. Shinbrot and Muzzio observed an oscillating motion of the intruder on the top, which corresponds to the "whale effect" predicted by Pöschel and Herrmann [16]. Also they claim a "reverse buoyancy" in shaken granular beds. Möbius et al., analyzed the segregation effect in the presence of air and the interplay between vibration-induced convection and fluidization. They reported the intruder rising time dependence on density. Liffman et al. [10], analyzed the intruder ascent speed dependence on density and shaking frequency. Recently, the interplay between the intruder's size and material density have been considered by Hong et al. [22]. They propose a phase diagram for the upward/downward intruder's movement. Ohtsuki et al., performed molecular dynamics simulations in two dimensions and studied the effect of intruder size and density on the height, and found no segregation threshold [17]. Recently Shishoda and Wassgren performed two dimensional simulations to model segregation in vibrofluidized beds [23]. They reported an height dependence with the density ratio between the intruder and the surrounding particles. In their model the intruder position result from a balance between the granular pressure (buoyant force) within the bed and the intruder weight. Their approach is in some sense similar to the model that we propose in this article.

Subject to an external force, granular materials locally perform random motions as a result of collisions between grains, much like the molecules in a gas. This picture has inspired several authors to use kinetic theories to derive continuum equations for the granular flow-field variables [28-34]. Some of these theories have been generalized to multicomponent mixtures of grains [35-38]. For different size particles in the presence of a temperature gradient, Arnarson and Willits, found that larger, denser particles tend to be more concentrated in cooler regions [37]. This result was confirmed by numerical simulations $[39,40]$. However, this mechanism of segregation is a natural consequence of the imposed gradient of temperature and its not related to the nature of the grains $[40]$.

In this article we address the problem of size segregation using a kinetic theory approach in two and three dimensions $(D=2,3)$. We consider the case of an intruder particle immersed in a granular bed. We assume that the material density of all particles is the same. We propose a segregation mechanism based on the difference of densities between different regions of the system, which give 
origin to a buoyant force that acts on the intruder. The difference of densities is caused by the difference between the mean kinetic energy among the region around the intruder and the medium without intruder. The dissipative nature of the collisions between the particles of a granular media is responsible for this mean energy difference, and modifies the mobility of the particles.

The plan of this article is as follows. In Section 2, we derive a continuum formulation for the granular fluid, and introduce the definition of the "granular temperature". In Section 3, we propose an analytic method to estimate the local temperature in the system. In Section 4, we introduce the coefficient of thermal expansion. In Section 5, explicit solutions of the time dependence of height and velocity of the large particle are calculated. We can explain the size ratio dependence of the rise velocity and address the issue of the critical size ratio to segregation. To validate our arguments we make comparisons with previous experimental data.

\section{Continuum formulation}

We consider an intruder particle of mass $m_{I}$ and radius $r_{I}$ immersed in a granular bed. The granular bed is formed of $N$ monodisperse particles of mass $m_{F}$ and radius $r_{F}$. The particles are modeled by inelastic hard disks $(D=2)$ or spheres $(D=3)$ in a $D$-dimensional volume $V=L^{D}$ of size $L$. The size ratio is denoted $\phi=r_{I} / r_{F}$. The particles interact via binary encounters. The inelasticity is specified by a restitution coefficient $e \leq 1$. We assume this restitution coefficient to be a constant, independent on the impact velocity and the same for the fluid particles and the intruder. The post collisional velocities $\mathbf{v}^{\prime}$ are given in terms of the pre-collisional velocities $\mathbf{v}$ by

$$
\mathbf{v}_{1,2}^{\prime}=\mathbf{v}_{1,2} \mp \frac{m_{r e d}(1+e)}{m_{1,2}}\left[\left(\mathbf{v}_{1}-\mathbf{v}_{2}\right) \cdot \hat{\mathbf{n}}\right] \hat{\mathbf{n}}
$$

where the labels 1 or 2 specify the particle, $\hat{\mathbf{n}}$ is the unit vector normal to the tangential contact plane pointing from 1 to 2 at the contact time, and the reduced mass $m_{\text {red }}=m_{1} m_{2} /\left(m_{1}+m_{2}\right)$. To calculate the dissipated energy we consider that energy is dissipated only by collisions between pairs of grains. In a binary collision the energy dissipated is proportional to $\Delta E=-m_{\text {red }}(1-$ $\left.e^{2}\right) v^{2} / 2$, where $v$ is the mean velocity of the particles.

In this work we use a generalized notion of temperature. In a vibrofluidized granular material a "granular temperature" $T_{g}$ can be defined to describe the random motion of the grains and is the responsible for the pressure, and the transport of momentum and energy in the system [31]. The granular temperature $T_{g}$ is defined proportional to the mean kinetic energy $E$ associated to 
the velocity of each particle

$$
\frac{D}{2} T_{g}=\frac{E}{N}=\frac{1}{N} \sum_{i=1}^{N}\left(\frac{1}{2} m_{i} v_{i}^{2}\right)
$$

We expect a continuum limit to hold for $N \gg 1$, when the small particles may be considered as forming a granular fluid [41]. In order to develop an analytic study, we assume that the uniformly heated granular fluid can be described by the standard hydrodynamic equations [41] derived from kinetic theories for granular systems [28-34]. In this study, we focus on a steady state with no macroscopic flow.

The balance equation for the energy is

$$
\nabla \cdot \mathbf{q}=-\gamma
$$

where $\mathbf{q}$ is the flux of energy and $\gamma$ is the average rate of dissipated energy due to the inelastic nature of the particles collisions. The constitutive relation for the flux of energy,

$$
\mathbf{q}=-\kappa \nabla T_{g}
$$

defines the thermal conductivity $\kappa$. Consequently, we have

$$
\nabla \cdot\left(\kappa \nabla T_{g}\right)=\gamma
$$

An uniformly fluidized state can be realized when the granular material is vibrated in the vertical direction, typically as $z_{0}(t)=A_{0} \sin \left(\omega_{0} t\right)$, with the amplitude $A_{0}$ and the frequency $\omega_{0}=2 \pi f$, so that one can define a typical velocity $u_{0}=A_{0} \omega_{0}$. In the experiments the excitation is described by the dimensionless acceleration $\Gamma_{0}=A_{0} \omega_{0}^{2} / \mathrm{g}$, where $\mathrm{g}$ is the gravitational acceleration. As a first approximation the effect of the external force experienced by the fluid particles due to the gravitational field is neglected in the description of the granular flow. Experimentally this correspond to the regime $\Gamma_{0} \gg 1$. So, the momentum balance, in the steady state, implies that the pressure $p$ is constant throughout the system.

The hydrodynamic equations close with the state equation, the collisional dissipation $\gamma$ and the transport coefficients for a granular medium. In the limit $N \gg 1$ the constitutive relations are determined as function of the properties of the small grains. The transport coefficients are assumed to be given by the Enskog theory for dense gases in the limit of small inelasticity. 
The total pressure should be essentially equal to that of the small particles, the contribution of the intruder being negligible, since $N \gg 1$. For a dense system the pressure is related to the density by the virial equation of state, which in the case of inelastic particles is $[28,34]$

$$
p=n T_{g}[1+(1+e)(D-1) G]
$$

where $n=N / V$ is the number density and $G=\nu g_{0}$, where $\nu=\Omega_{D} n r_{F}^{D} / D$ is the volume fraction, with $\Omega_{D}=2 \pi^{D / 2} / \Gamma(D / 2)$ as the surface area of a $D$ dimensional unit sphere and $g_{0}$ is the pair correlation function. For disks $g_{0}$ is taken to be that proposed by Verlet and Levesque [42]:

$$
g_{0}=\frac{1}{1-\nu}+\frac{9}{16} \frac{\nu}{(1-\nu)^{2}}
$$

with the area fraction $\nu=n \pi r_{F}^{2}$, and that proposed by Carnahan and Starling for spheres [43]:

$$
g_{0}=\frac{1}{1-\nu}+\frac{\nu}{2(1-\nu)^{2}}\left[3+\frac{\nu}{2(1-\nu)}\right]
$$

with the volume fraction $\nu=4 \pi n r_{F}^{3} / 3$.

It is important to mention that the equations (7) and (8) only work for moderate densities. In Ref. [44] Luding and Strauß showed that $g_{0}$ should diverge at the close packing limit $\left(\nu=\nu_{\max }\right)$, rather than at $\nu \rightarrow 1$. Therefore, in the present model we assume that $\nu<\nu_{\max }$.

The state-dependent thermal conductivity possesses the general form [28,32]

$$
\kappa=\kappa_{0} \sqrt{T_{g}}
$$

where the prefactor $\kappa_{0}$ is a function of the fluid particle properties, and can be calculated using a Chapman-Enskog procedure through the solution of Enskog transport equation $[28,32,34,45]$. The explicit expressions of these prefactors are given in Appendix A.

To estimate the collisional dissipation rate $\gamma$ we consider the loss of average kinetic energy per collision and per unit time. In a binary collision the kinetic energy dissipated can be expressed in terms of the granular temperature as $\Delta E=-\left(1-e^{2}\right) T_{g} / 2$. For the fluid particles, the average collision frequency $\omega_{F}$ is proportional to $\omega_{F} \sim \sqrt{T_{g}}$, and we assume that it is given by the Enskog 
collision frequency [45]

$$
\omega_{F}=\frac{\Omega_{D}}{\sqrt{2 \pi}} n g_{0}\left(2 r_{F}\right)^{D-1}\left(\frac{2}{m_{F}}\right)^{1 / 2} T_{g}^{1 / 2} .
$$

This form for the frequency of collisions is justified for a granular medium. This is a consequence that the average spacing between nearest neighbor $s$ is supposed to be less than the grain diameter $\left(s \ll 2 r_{F}\right)$ [41]. Multiplying $\Delta E$ by the collision rate $\omega_{F}$ and the number density $n=N / V$, we obtain the collisional dissipation rate $\gamma_{F}$ for the fluid particles

$$
\gamma_{F}=\frac{\Omega_{D}}{2 \sqrt{2 \pi}}\left(1-e^{2}\right) n^{2} g_{0}\left(2 r_{F}\right)^{D-1}\left(\frac{2}{m_{F}}\right)^{1 / 2} T_{g}^{3 / 2} .
$$

In order to simplify the mathematical notation let us express $\gamma_{F}$ as

$$
\gamma_{F}=\xi_{F} T_{g}^{3 / 2}
$$

where the dissipation factor $\xi_{F}$ contains the prefactors which multiply $T_{g}^{3 / 2}$ in Eq.(11), this is

$$
\xi_{F} \equiv \frac{\Omega_{D}}{2 \sqrt{2 \pi}}\left(1-e^{2}\right) n^{2} g_{0}\left(2 r_{F}\right)^{D-1}\left(\frac{2}{m_{F}}\right)^{1 / 2}
$$

To understand the essential features of the intruder's presence in the granular medium, it is adequate to adopt a simplified point of view. If the mean velocity of the fluid particles is $v \propto \sqrt{T_{g} / m}$, the flux of fluid particles which strikes the intruder's surface can be estimate as $n v$. Multiplying this flux by the area of the intruder $\Omega_{D} r_{I}^{(D-1)}$, we can calculate the number of fluid particles which strike the surface of the intruder per unit time, and written in terms of the granular temperature we have

$$
\omega_{I}=\frac{\Omega_{D}}{\sqrt{2 \pi}} n \tilde{g}_{0}\left(r_{F}+r_{I}\right)^{D-1}\left(\frac{m_{I}+m_{F}}{m_{I} m_{F}}\right)^{1 / 2} T_{g}^{1 / 2}
$$

where $\tilde{g}_{0}$ is the pair correlation function of the granular fluid in presence of the intruder.

The local density of kinetic energy dissipated in the region near the intruder is

$$
\gamma_{I}=\frac{\Omega_{D}}{2 \sqrt{2 \pi}}\left(1-e^{2}\right) \frac{n}{V} \tilde{g}_{0}\left(r_{F}+r_{I}\right)^{D-1}\left(\frac{m_{I}+m_{F}}{m_{I} m_{F}}\right)^{1 / 2} T_{g}^{3 / 2} .
$$


In a simplified form Eq.(15) can be expressed as

$$
\gamma_{I}=\xi_{I} T_{g}^{3 / 2}
$$

where the dissipation factor $\xi_{I}$ is defined as

$$
\xi_{I} \equiv \frac{\Omega_{D}}{2 \sqrt{2 \pi}}\left(1-e^{2}\right) \frac{n}{V} \tilde{g}_{0}\left(r_{F}+r_{I}\right)^{D-1}\left(\frac{m_{I}+m_{F}}{m_{I} m_{F}}\right)^{1 / 2} .
$$

Finally, in order to estimate $\gamma_{I}$ an explicit expression for $\tilde{g}_{0}$ is needed. To our knowledge, no theory exists for the exact calculation of the pair correlation function of a single intruder (tracer particle) immersed in a granular fluid. Rather than going into details concerning the calculation of $\tilde{g}_{0}$, we propose to use the generalization of equations (7) and (8) for binary mixtures $[46,47]$, restricted in our case to the limit where $N>>1$ and $r_{I}>r_{F}$. For two dimensions we can verify that

$$
\tilde{g}_{0} \simeq \frac{1}{1-\nu}+\frac{9}{8} \frac{\nu}{(1-\nu)^{2}}
$$

and for three dimensions

$$
\tilde{g}_{0} \simeq \frac{1}{1-\nu}+\frac{\nu}{(1-\nu)^{2}}\left[3+\frac{\nu}{1-\nu}\right]
$$

Let us remark that $g_{0}$ and $\tilde{g}_{0}$ are not very different and both quantities tend to 1 in the diluted limit. Here too, we assume that $\nu<\nu_{\max }$.

\section{Local temperature difference}

The intruder's presence modifies the local temperature of the system due to the collisions that happen at its surface. The number of collisions on the surface increases with the size of the particle, but the local density of dissipated energy diminishes. From Eq.(5) we can calculate within a sphere of radius $r_{0}$ the value of the temperature in the granular fluid in presence of the intruder and compare it with the temperature in the granular fluid without intruder, we will denote these temperatures $T_{1}$ and $T_{2}$ respectively (see Fig.(1)). These spherical regions are considered to be placed in the reference frame of the intruder particle. This is a simple method to estimate the temperature difference between a region with intruder and a region without intruder $\Delta T_{g}=T_{1}-T_{2}$. Let us concentrate on solutions with radial symmetry. The solutions of Eq.(5), 


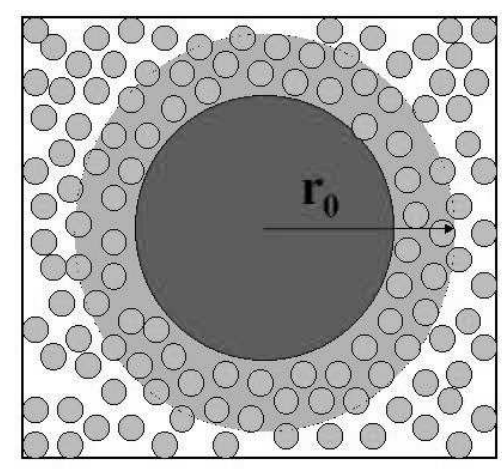

(a)

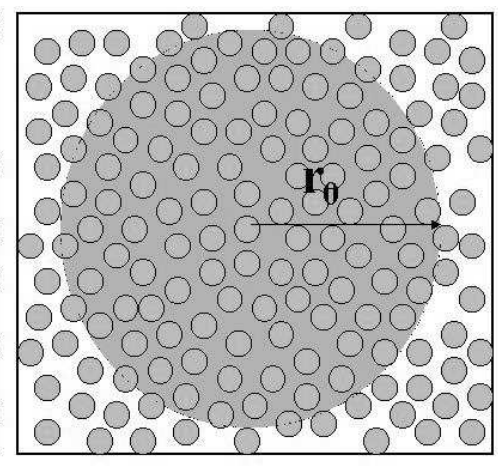

(b)

Fig. 1. Schematic representation of the regions used to calculate the granular temperature. (a) Region around the intruder within a sphere of radius $r_{0}$ and (b) region without intruder.

for an arbitrary dimension $D$, satisfy the equation

$$
\frac{1}{r^{D-1}} \frac{d}{d r}\left(r^{D-1} \kappa_{0} T_{g}^{1 / 2} \frac{d T_{g}}{d r}\right)=\xi T_{g}^{3 / 2} .
$$

This nonlinear differential equation can be simplified by the fact that the pressure is considered constant throughout the system and remembering that $p \sim T_{g}$. So, linearizing Eq.(20) the resulting equation may be written in terms of $w \equiv T_{g}^{1 / 2}$,

$$
\frac{d^{2} w}{d r^{2}}+\frac{D-1}{r} \frac{d w}{d r}=\lambda^{2} w
$$

where

$$
\lambda^{2} \equiv \frac{\xi}{2 \kappa_{0}}
$$

It is useful at this point to see the implications of the requirement of constant pressure invoked to derive Eq.(21). This estimate is suggested by the fact that the effect of the gravitational field can be neglected in the regime of strong perturbations. Then, the momentum balance equation for fluid particles satisfies $\nabla p=0$. This is a good approximation if the kinetic energy is much greater than the change of the gravitational potential energy experienced over the average spacing between grains. However, we can not forget that, the granular fluid is a system out of "thermal" and mechanical equilibrium (See Section 4 bellow). A change in the local granular temperature does change the pressure of the system. To make the model analytically tractable, we have neglected all the gradients of the pressure. This heuristic assumption is based on the 
fact that a dense granular fluid can be considered as an incompressible system [41]. Qualitatively, this picture is correct if the "isothermal compressibility" $k_{p}$ (See Sec. 4 Eq.(58)) is bigger than the coefficient of "thermal expansion" $\alpha$ (See Sec. 4 Eq.(57)). To be more concrete, using the thermodynamic relation $(\partial p / \partial T)_{V}=\alpha / k_{p}$, it follows that if $k_{p}>>\alpha$, then $\partial p / \partial T \simeq 0$.

Therefore, in the present model, the gradients $\partial p / \partial T$ and $\nabla p$ do not contribute to the description of the granular fluid. The effect of $g$ must be included in the description of the system at the level of individual grains. These rough approximations, which should be sufficiently accurate for our purpose, might not always be valid. It is necessary to be aware that in proceeding along this way some part of the dynamics may be lost. For example, a complete analysis of Eq.(20) requires much more information, such as the behavior of "compressive waves" arising from the variations of the pressure, which drives the system toward the mechanical equilibrium.

The collisional dissipation rate can be decomposed in two parts. We propose this decomposition supposing that the energy dissipation around the intruder is dominated by the collisions between the small grains and the intruder, then the dissipation rate in this region is given by Eq.(15). In the rest of the system the dissipation rate is dominated by the collisions between small grains only. In this case the dissipation is given by Eq.(11).

First, let us consider the "inhomogeneous case" when the intruder is localized in the center of the system $(r=0)$, see Fig.(1a). The dissipation factor $\xi$ can be decomposed in two parts: $\xi=\xi_{I}$ for the region near the intruder $\left(r=r_{I}\right)$, and $\xi=\xi_{F}$ for the region $\left(r_{I}<r \leq r_{0}\right)$, where $r_{0}$ is the radius of the considered region.

For the inhomogeneous case we express Eq.(21) as

$$
\frac{d^{2} w}{d r^{2}}+\frac{D-1}{r} \frac{d w}{d r}=\left\{\begin{array}{l}
\lambda_{I}^{2} w \text { for } 0<r \leq r_{I}, \\
\lambda_{F}^{2} w \text { for } r_{I}<r \leq r_{0},
\end{array}\right.
$$

where, $\lambda_{I}^{2} \equiv \xi_{I} / 2 \kappa_{0}$ and $\lambda_{F}^{2} \equiv \xi_{F} / 2 \kappa_{0}$. The solution of Eq.(23) is determined by the boundary condition imposed upon the system. As boundary condition we suppose that the system is enclosed by an external surface of radius $r_{0}$ at temperature $T_{g}\left(r_{0}\right)=T_{0}$ (respectively, $\left.w\left(r_{0}\right)=w_{0}\right)$.

Let us denote $T_{1-}(r)$ the granular temperature for the region $\left(0<r \leq r_{I}\right)$, and $T_{1+}(r)$ the granular temperature for the region $\left(r_{I}<r \leq r_{0}\right)$ (respectively, $w_{-}(r) \equiv \sqrt{T_{1-}(r)}$ and $\left.w_{+}(r) \equiv \sqrt{T_{1+}(r)}\right)$. The intruder's presence imposes 
internal boundary conditions. On the inner surface, the temperature should satisfy

$$
\left.w_{-}(r)\right|_{r=r_{I}}=\left.w_{+}(r)\right|_{r=r_{I}} \text {. }
$$

The flux of energy also imposes another internal boundary condition. If we suppose the flux of energy continuous on the inner surface, from Eq.(4) the granular temperature should satisfy

$$
\left.\frac{d w_{-}(r)}{d r}\right|_{r=r_{I}}=\left.\frac{d w_{+}(r)}{d r}\right|_{r=r_{I}} .
$$

\subsection{Solution for $2 D$}

The solutions to Eq.(23) for $D=2$ are a linear combination of the modified Bessel function of order zero $w_{1}(r)=\left\{I_{0}(\lambda r), K_{0}(\lambda r)\right\}$ [48]. The general solution is

$$
w_{-}(r)=A_{-} I_{0}\left(\lambda_{I} r\right)+B_{-} K_{0}\left(\lambda_{I} r\right) \quad \text { for } 0<r \leq r_{I},
$$

and

$$
w_{+}(r)=A_{+} I_{0}\left(\lambda_{F} r\right)+B_{+} K_{0}\left(\lambda_{F} r\right) \quad \text { for } r_{I}<r \leq r_{0},
$$

where $A_{-}, A_{+}, B_{-}$and $B_{+}$are constants that must be determined from the boundary conditions.

The function $K_{0}(\lambda r)$ diverges when $r \rightarrow 0$, then

$$
B_{-}=0
$$

When $r=r_{0}$ the Eq.(27) should satisfy the boundary condition

$$
\left.w_{+}(r)\right|_{r=r_{0}}=w_{0}
$$

this is,

$$
A_{+} I_{0}\left(\lambda_{F} r_{0}\right)+B_{+} K_{0}\left(\lambda_{F} r_{0}\right)=w_{0}
$$

On the inner surface the boundary condition $(24) w_{-}\left(r_{I}\right)=w_{+}\left(r_{I}\right)$ leads to 


$$
\begin{aligned}
A_{-} I_{0}\left(\lambda_{I} r_{I}\right) & =A_{+} I_{0}\left(\lambda_{F} r_{I}\right)+B_{+} K_{0}\left(\lambda_{F} r_{I}\right) \\
\Longrightarrow A_{-} & =A_{+} \frac{I_{0}\left(\lambda_{F} r_{I}\right)}{I_{0}\left(\lambda_{I} r_{I}\right)}+B_{+} \frac{K_{0}\left(\lambda_{F} r_{I}\right)}{I_{0}\left(\lambda_{I} r_{I}\right)}
\end{aligned}
$$

The inner boundary condition (25) leads to

$$
\begin{aligned}
A_{-} \lambda_{I} I_{1}\left(\lambda_{I} r_{I}\right) & =A_{+} \lambda_{F} I_{1}\left(\lambda_{F} r_{I}\right)-B_{+} \lambda_{F} K_{1}\left(\lambda_{F} r_{I}\right) \\
\Longrightarrow A_{-} & =\left(\frac{\lambda_{F}}{\lambda_{I}}\right)\left[A_{+} \frac{I_{1}\left(\lambda_{F} r_{I}\right)}{I_{1}\left(\lambda_{I} r_{I}\right)}-B_{+} \frac{K_{1}\left(\lambda_{F} r_{I}\right)}{I_{1}\left(\lambda_{F} r_{I}\right)}\right] .
\end{aligned}
$$

Equating Eqs.(31) and (32) we find

$$
\begin{aligned}
\frac{A_{+}}{B_{+}} & =\frac{\lambda_{F} I_{0}\left(\lambda_{I} r_{I}\right) K_{1}\left(\lambda_{F} r_{I}\right)+\lambda_{I} I_{1}\left(\lambda_{I} r_{I}\right) K_{0}\left(\lambda_{F} r_{I}\right)}{\lambda_{F} I_{0}\left(\lambda_{I} r_{I}\right) I_{1}\left(\lambda_{F} r_{I}\right)-\lambda_{I} I_{1}\left(\lambda_{I} r_{I}\right) I_{0}\left(\lambda_{F} r_{I}\right)}, \\
& \equiv \Theta_{A B}
\end{aligned}
$$

From Eqs. (30) and (33) the constant $B_{+}$should be

$$
B_{+}=\frac{w_{0}}{\Theta_{A B} I_{0}\left(\lambda_{F} r_{0}\right)+K_{0}\left(\lambda_{F} r_{0}\right)} .
$$

Substituting Eq.(34) into (33) we have

$$
A_{+}=\frac{w_{0} \Theta_{A B}}{\Theta_{A B} I_{0}\left(\lambda_{F} r_{0}\right)+K_{0}\left(\lambda_{F} r_{0}\right)} .
$$

Substituting Eqs.(34) and (35) into (31) we have

$$
A_{-}=\frac{\Theta_{A B} I_{0}\left(\lambda_{F} r_{I}\right)+K_{0}\left(\lambda_{F} r_{I}\right)}{\Theta_{A B} I_{0}\left(\lambda_{F} r_{0}\right)+K_{0}\left(\lambda_{F} r_{0}\right)}\left(\frac{w_{0}}{I_{0}\left(\lambda_{I} r_{I}\right)}\right) .
$$

The granular temperature in the inhomogeneous case is

$$
T_{1}(r)= \begin{cases}\left(A_{-} I_{0}\left(\lambda_{I} r\right)\right)^{2} & \text { for } 0<r \leq r_{I}, \\ \left(A_{+} I_{0}\left(\lambda_{F} r\right)+B_{+} K_{0}\left(\lambda_{F} r\right)\right)^{2} & \text { for } r_{I}<r \leq r_{0},\end{cases}
$$

where the constant $A_{-}, A_{+}$and $B_{+}$are given by the Eqs.(36), (35) and (34), respectively. 
In the "homogeneous case", see Fig.(1b), the prefactor $\lambda_{I}=0$. Then the granular temperature $T_{2}(r)$ is

$$
T_{2}(r)=\left(\frac{I_{0}\left(\lambda_{F} r\right)}{I_{0}\left(\lambda_{F} r_{0}\right)}\right)^{2} T_{0}
$$

Now we are interested in determining the temperature difference $\Delta T_{g}$ between case 1 and 2 in the granular fluid. For this we calculate the granular temperatures at $r=r_{I}$. So, Eq.(37) and (38) lead to

$$
\begin{gathered}
T_{1}\left(r_{I}\right)=\left(\frac{\Theta_{A B} I_{0}\left(\lambda_{F} r_{I}\right)+K_{0}\left(\lambda_{F} r_{I}\right)}{\Theta_{A B} I_{0}\left(\lambda_{F} r_{0}\right)+K_{0}\left(\lambda_{F} r_{0}\right)}\right)^{2} T_{0}, \\
T_{2}\left(r_{I}\right)=\left(\frac{I_{0}\left(\lambda_{F} r_{I}\right)}{I_{0}\left(\lambda_{F} r_{0}\right)}\right)^{2} T_{0} .
\end{gathered}
$$

Then, the temperature difference is

$$
\Delta T_{g}=\left[\left(\frac{\Theta_{A B} I_{0}\left(\lambda_{F} r_{I}\right)+K_{0}\left(\lambda_{F} r_{I}\right)}{\Theta_{A B} I_{0}\left(\lambda_{F} r_{0}\right)+K_{0}\left(\lambda_{F} r_{0}\right)}\right)^{2}-\left(\frac{I_{0}\left(\lambda_{F} r_{I}\right)}{I_{0}\left(\lambda_{F} r_{0}\right)}\right)^{2}\right] T_{0}
$$

in two dimensions.

\subsection{Solution for 3D}

When $D=3$, the solution of Eq.(23) is given in terms of the spherical modified Bessel functions of zero order $w_{1}(r)=\left\{i_{0}(\lambda r)=\sinh (\lambda r) / \lambda r, k_{0}(\lambda r)=\right.$ $\left.e^{-\lambda r} / \lambda r\right\}[48]$. The general solution in this case is

$$
w_{-}(r)=A_{-} i_{0}\left(\lambda_{I} r\right)+B_{-} k_{0}\left(\lambda_{I} r\right) \quad \text { for } 0<r \leq r_{I},
$$

and

$$
w_{+}(r)=A_{+} i_{0}\left(\lambda_{F} r\right)+B_{+} k_{0}\left(\lambda_{F} r\right) \quad \text { for } r_{I}<r \leq r_{0}
$$

The function $k_{0}(\lambda r)$ diverges when $r \rightarrow 0$, then

$$
B_{-}=0
$$

The constants $A_{-}, A_{+}$and $B_{+}$, are calculated from the boundary conditions in a similar way as before: 


$$
\begin{aligned}
& A_{-}=\frac{\Theta_{A B} i_{0}\left(\lambda_{F} r_{I}\right)+k_{0}\left(\lambda_{F} r_{I}\right)}{\Theta_{A B} i_{0}\left(\lambda_{F} r_{0}\right)+i_{0}\left(\lambda_{F} r_{0}\right)}\left(\frac{w_{0}}{i_{0}\left(\lambda_{I} r_{I}\right)}\right), \\
& A_{+}=\frac{w_{0} \Theta_{A B}}{\Theta_{A B} i_{0}\left(\lambda_{F} r_{0}\right)+k_{0}\left(\lambda_{F} r_{0}\right)}, \\
& B_{+}=\frac{w_{0}}{\Theta_{A B} i_{0}\left(\lambda_{F} r_{0}\right)+k_{0}\left(\lambda_{F} r_{0}\right)},
\end{aligned}
$$

where in this case the factor $\Theta_{A B}$ is

$$
\Theta_{A B}=\frac{\lambda_{F} i_{0}\left(\lambda_{I} r_{I}\right) k_{1}\left(\lambda_{F} r_{I}\right)+\lambda_{I} i_{1}\left(\lambda_{I} r_{I}\right) k_{0}\left(\lambda_{F} r_{I}\right)}{\lambda_{F} i_{0}\left(\lambda_{I} r_{I}\right) i_{1}\left(\lambda_{F} r_{I}\right)-\lambda_{I} i_{1}\left(\lambda_{I} r_{I}\right) i_{0}\left(\lambda_{F} r_{I}\right)} .
$$

The granular temperature in the inhomogeneous case in $3 \mathrm{D}$ is

$$
T_{1}(r)= \begin{cases}\left(A_{-} i_{0}\left(\lambda_{I} r\right)\right)^{2} & \text { for } 0<r \leq r_{I}, \\ \left(A_{+} i_{0}\left(\lambda_{F} r\right)+B_{+} k_{0}\left(\lambda_{F} r\right)\right)^{2} & \text { for } r_{I}<r \leq r_{0},\end{cases}
$$

where the constant $A_{-}, A_{+}$and $B_{+}$are given by the Eqs.(45), (46) and (47). In the "homogeneous case" the prefactor $\lambda_{I}=0$. Then the granular temperature $T_{2}(r)$ is

$$
T_{2}(r)=\left(\frac{i_{0}\left(\lambda_{F} r\right)}{i_{0}\left(\lambda_{F} r_{0}\right)}\right)^{2} T_{0}
$$

Again the temperature difference $\Delta T_{g}$ between case 1 and 2 is calculated at $r=r_{I}$,

$$
\begin{gathered}
T_{1}\left(r_{I}\right)=\left(\frac{\Theta_{A B} i_{0}\left(\lambda_{F} r_{I}\right)+k_{0}\left(\lambda_{F} r_{I}\right)}{\Theta_{A B} i_{0}\left(\lambda_{F} r_{0}\right)+k_{0}\left(\lambda_{F} r_{0}\right)}\right)^{2} T_{0}, \\
T_{2}\left(r_{I}\right)=\left(\frac{i_{0}\left(\lambda_{F} r_{I}\right)}{i_{0}\left(\lambda_{F} r_{0}\right)}\right)^{2} T_{0} .
\end{gathered}
$$

Then, the temperature difference is

$$
\Delta T_{g}=\left[\left(\frac{\Theta_{A B} i_{0}\left(\lambda_{F} r_{I}\right)+k_{0}\left(\lambda_{F} r_{I}\right)}{\Theta_{A B} i_{0}\left(\lambda_{F} r_{0}\right)+k_{0}\left(\lambda_{F} r_{0}\right)}\right)^{2}-\left(\frac{i_{0}\left(\lambda_{F} r_{I}\right)}{i_{0}\left(\lambda_{F} r_{0}\right)}\right)^{2}\right] T_{0}
$$

in three dimensions. 


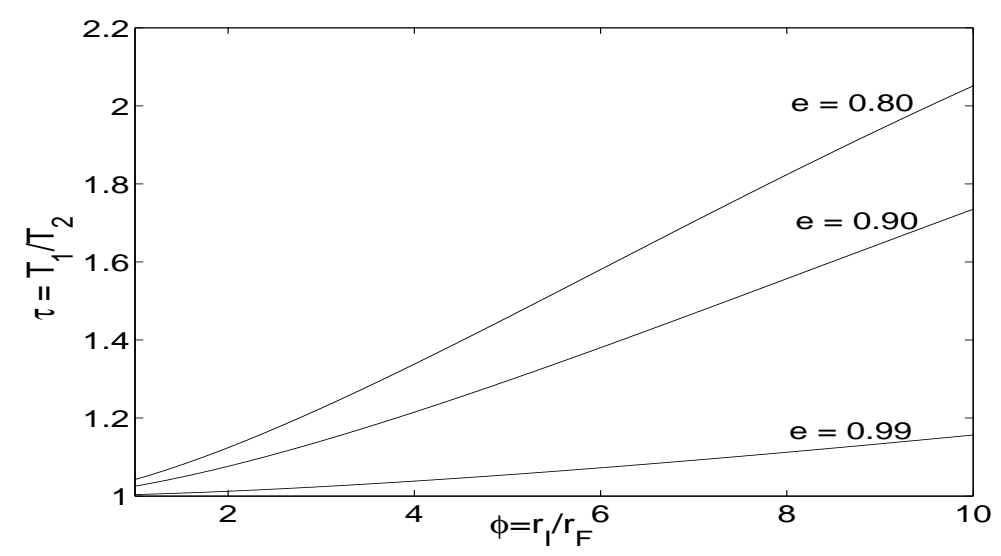

Fig. 2. Ratio $\tau=T_{1} / T_{2}$ of the granular temperatures, showing non-equipartition of energy $(\tau \neq 1)$ for different values of the coefficient of restitution $e$.

\subsection{Energy equipartition breakdown}

Let us define the temperature ratio $\tau \equiv T_{1} / T_{2}$. In two dimension we have

$$
\tau=\left(\frac{I_{0}\left(\lambda_{F} r_{0}\right)\left[\Theta_{A B} I_{0}\left(\lambda_{F} r_{I}\right)+K_{0}\left(\lambda_{F} r_{I}\right)\right]}{I_{0}\left(\lambda_{F} r_{I}\right)\left[\Theta_{A B} I_{0}\left(\lambda_{F} r_{0}\right)+K_{0}\left(\lambda_{F} r_{0}\right)\right]}\right)^{2},
$$

and for three dimensions,

$$
\tau=\left(\frac{i_{0}\left(\lambda_{F} r_{0}\right)\left[\Theta_{A B} i_{0}\left(\lambda_{F} r_{I}\right)+k_{0}\left(\lambda_{F} r_{I}\right)\right]}{i_{0}\left(\lambda_{F} r_{I}\right)\left[\Theta_{A B} i_{0}\left(\lambda_{F} r_{0}\right)+k_{0}\left(\lambda_{F} r_{0}\right)\right]}\right)^{2}
$$

since $\lambda_{F}>\lambda_{I}$ we can verify that $T_{1}>T_{2}$, this means $\tau>1$. So, the temperatures ratio between the region with intruder and the region without intruder are different. In our model this lack of equipartition is due to a difference between the collisional dissipation rate related to the particle sizes. In the elastic limit $e \rightarrow 1$ the energy equipartition is restored $\tau \rightarrow 1$. In Fig. 2, we present the qualitative behavior of $\tau$ with the size ratio $\phi=r_{I} / r_{F}$, for different values of the coefficient $e$. The granular temperature difference increases with $\phi$ and depends on $e$. We can see that $\tau$ is nearly constant and very close to unity when $e=0.99$.

Recently, this quantity was directly measured in experiments performed by Wildman and Parker [49] and Feitosa and Menon [50]. They observed that energy equipartition does not generally hold for a binary vibrated granular system. They reported that the ratio of granular temperatures depends on the ratio of particle mass densities. Also in fluidized binary granular mixtures the breakdown of energy equipartition was observed experimentally [51] and described theoretically in the framework of the kinetic theory [52]. Certainly 
these experiments don't correspond to the typical conditions for size segregation experiments, but they support the idea of a temperature difference in the system due to the presence of the intruder particle. The experimental results reported by Wildman and Parker, show that the granular temperature of the larger particles was higher than that of the smaller particles, this evidence supports the new picture proposed in this work.

\section{Thermal expansion}

Granular materials are non-equilibrium systems and certainly they can not be considered ergodic in the traditional sense. The system increases its energy as a result of external driving (e.g., vibration) while its decreases its energy by dissipation. There have been different attempts to define a statistical mechanics for granular media [53-55]. Recent studies suggest that the thermodynamic description proposed by Edwards [54] opens a door towards a statistical description of compact granular matter [56]. However, these findings are for weak driving and the generalization to stronger forcing is not evident.

The thermodynamic formulation proposed by Herrmann (See Ref. [53] for a detailed discussion) starts from the energy flux balance and the analog for the "equilibrium" is a steady state driven by the energy flux. If one allows for changes in the volume of the system the energy conservation will become $\Delta I=\Delta E_{\text {int }}+\Delta D+\Delta W$, where $\Delta I$ is the energy that was pumped into the system in a given time, $\Delta E_{\text {int }}$ is the change of "internal energy" (e.g., kinetic energy), $\Delta D$ is the energy dissipated in a given time and $\Delta W$ is the work done to change the volume. Theoretically we can derive the energy relaxation to the steady state for a driven granular medium [57]. The system can be considered to be in "equilibrium" when the excess of dissipated energy $(\Delta \tilde{D}=\Delta I-\Delta D)$ should be zero [53]. Under this theoretical assumption and in the framework of the kinetic theory we proceed to calculate the thermal expansion coefficient for a granular fluid.

The change of mean energy of the system is basically due to a mechanical interaction with their external parameters (e.g., the amplitude $A_{0}$ and the frequency $\omega_{0}=2 \pi f$ of vibration, the volume of the system $V$, and the pressure $p$ ). The work $W$ done to change the volume of the system from $V$ to a certain quantity $V+d V$ is equal to the change of its mean energy and its related to the mean pressure and volume by $d W=p d V+V d p$. From the definition

of granular temperature, the change of the granular temperature depends on the mean kinetic energy of the particles. A volume change $d V$ is related to a temperature change $d T_{g}$ by the equation of state (6).

We can express $V$ as a function of $T_{g}$ and $p, V=V\left(T_{g}, p\right)$. Thus given in- 
finitesimal changes in $T_{g}$ and $p$, we can write

$$
\begin{aligned}
d V & =\left(\frac{\partial V}{\partial T_{g}}\right)_{p} d T_{g}+\left(\frac{\partial V}{\partial p}\right)_{T_{g}} d p \\
& =\alpha V d T_{g}-k_{p} V d p
\end{aligned}
$$

where $\alpha$ is the thermal expansion coefficient defined as

$$
\alpha \equiv \frac{1}{V}\left(\frac{\partial V}{\partial T_{g}}\right)_{p}=-\frac{1}{n}\left(\frac{\partial n}{\partial T_{g}}\right)_{p, N}
$$

and $k_{p}$ is the "isothermal compressibility" defined as

$$
k_{p} \equiv-\frac{1}{V}\left(\frac{\partial V}{\partial p}\right)_{T_{g}}=\frac{1}{n}\left(\frac{\partial n}{\partial p}\right)_{T_{g}} .
$$

If in a first approximation we neglect the variations of the coefficients $\alpha$ and $k_{p}$, we can integrate Eq.(56) and find

$$
\begin{aligned}
V\left(T_{g}, p\right) & =V_{0} \exp \left[\alpha \Delta T_{g}-k_{p} \Delta p\right], \\
& \approx V_{0}\left[1+\alpha \Delta T_{g}-k_{p} \Delta p\right] .
\end{aligned}
$$

Now, under the assumption of negligible compressibility, the density changes are caused by temperatures changes alone. From the temperature difference, Equations (41) and (53), the thermal expansion coefficient is

$$
\tilde{\rho}=\rho\left(1-\alpha \Delta T_{g}\right)
$$

Here, the constant density $\rho$ acts as a reference density corresponding to the reference temperature $T_{0}$, which can be taken to be the mean temperature in the flow. This is valid only in some average sense, when all the particles have the same density.

The thermal expansion coefficient can be derived from the equation of state (6) and definition (57). The general form of the coefficient of thermal expansion is

$$
\alpha=\frac{1}{T_{0}} C(\nu)
$$

where $C(\nu)$ is a correction due to the density of the system. In the dilute limit $\nu \rightarrow 0$ and $C(\nu) \rightarrow 1$, and the above expression tends to the expected value 
for a classical gas $\alpha=1 / T_{0}$. The explicit form of $C(\nu)$ is given in Appendix B.

\section{Segregation forces}

Buoyancy forces arise as a result of variations of density in a fluid subject to gravity. In the previous section we have introduced the change in the density of the granular fluid through the thermal expansion produced by the difference of granular temperatures calculated in Section 3. Now we propose that this density difference leads to a buoyancy force $\mathbf{f}_{b}$, similar to the Archimedean force

$$
\mathbf{f}_{b}=\Delta \rho V_{I} \mathbf{g}
$$

where $\Delta \rho=-\alpha \rho \Delta T_{g}, V_{I}=\frac{\Omega_{D}}{D} r_{I}^{D}$ is the $D$-dimensional volume of the intruder and $\mathbf{g}$ is the gravity field. Densities variations driven by granular temperature gradients due to inelastic collisions, were observed by Ramirez et al., [58] in molecular dynamic simulations, which in the presence of gravity, produces a buoyancy force driving the onset of convection cells. Recently, experimental evidence for this buoyancy-driven convection has been reported by Wildman et al., [59]. In our model the temperature gradient is obtained from the differences of the local density of dissipated energy between the region around the intruder and the region without intruder. The region with intruder is hotter than the region without intruder. This is a well established theoretical result shows in Section 3. It is important to note here that we are considering particles with equal material densities, so the buoyant force due to the material density differences $\mathbf{f}_{A}=\left(\rho_{F}-\rho\right) V_{I}$ g doesn't play any role in our analysis.

The intruder also experiences a viscous drag of the granular fluid. In the granular physics literature we find scarce studies of the forces on objects embedded inside granular flows [60,61]. Experiments performed by Zik et al., reported measurements of the mobility/friction coefficients of a sphere dragged horizontally through a vertically vibrated granular system. They observed a linear dependence of the drag force on the sphere velocity. If the resistive force $\mathbf{f}_{d}$ is either linear or quadratic in the velocity, the problem admits an analytical solution. In this work the drag force $\mathbf{f}_{d}$ is considered to be linear in the velocity of segregation $\mathbf{u}(t)$, analogous to the Stokes' drag force.

$$
\mathbf{f}_{d}=-6 \pi \mu r_{I} \mathbf{u}(t)
$$


where $\mu$ is the coefficient of viscosity of the granular fluid. The state-dependent viscosity possesses the general form $[28,32]$

$$
\mu=\mu_{0} \sqrt{T_{g}}
$$

where the prefactor $\mu_{0}$ is a function of the fluid particle properties, and can be calculated using a Chapman-Enskog procedure for the solution of Enskog transport equation. The explicit expressions of these prefactors are given in Appendix A.

Equation (63) is assumed to be valid for the particle Reynolds numbers $R e=$ $2 r_{F} \rho u / \mu$ less than unity. Calculating the settling velocity (see below) and the coefficient of viscosity of the granular fluid (calculated in Appendix A), we can show that $R e \sim 0$ and that the Stokes law assumption should be valid.

Equations (62) and (63) express the acting forces in the segregation process

$$
\mathbf{f}_{\text {seg }}=\mathbf{f}_{b}+\mathbf{f}_{d}
$$

Therefore, the equation of motion that governs the segregation process is

$$
\frac{\Omega_{D}}{D} r_{I}^{D} \rho \frac{d \mathbf{u}(t)}{d t}=-\frac{\Omega_{D}}{D} r_{I}^{D} \alpha \rho \Delta T_{g} \mathbf{g}-6 \pi \mu r_{I} \mathbf{u}(t)
$$

Now we suppose the granular system contained between two large parallel plates perpendicular to the gravitational field. We take the reference frame positive in the upward vertical direction. Arranging terms in Eq.(66) we find the following differential equation

$$
\frac{d u(t)}{d t}=\alpha \Delta T_{g} \mathrm{~g}-\frac{6 \pi D \mu\left(\phi r_{F}\right)^{1-D}}{\Omega_{D} \rho} u(t),
$$

where we have expressed the intruder's radius as function of the size ratio dependence $r_{I}=\phi r_{F}$, and the solution of this differential equation is the rise velocity of the intruder

$$
u(t)=\frac{\alpha \Delta T_{g} g t_{0}}{\phi^{1-D}}\left[1-\exp \left(-\phi^{1-D} \frac{t}{t_{0}}\right)\right]
$$

where the time-scale $t_{0}$ is given by

$$
t_{0} \equiv \frac{\Omega_{D} \rho}{6 \pi D \mu r_{F}^{1-D}} .
$$


The force balance between the drag force $\mathbf{f}_{d}$ and the buoyant force $\mathbf{f}_{b}$ gives the settling velocity $u_{s}$

$$
u_{s}=\frac{\alpha \Delta T_{g} g t_{0}}{\phi^{1-D}}
$$

The time dependent intruder height $z(t)$ is

$$
z(t)=\frac{\alpha \Delta T_{g} g t_{0}}{\phi^{1-D}}\left[t-t_{0} \phi\left(1-\exp \left(-\phi^{1-D} \frac{t}{t_{0}}\right)\right)\right]
$$

To estimate the granular temperature $T_{0}$, we adopt the scaling relationship between the granular temperature and the experimental parameter $A_{0}$ and $\omega_{0}$ proposed by Sunthar and Kumaran for dense vibrofluidized granular systems $[57]$ :

$$
T_{0}=\frac{2 \sqrt{2}}{\pi} \frac{m_{F} L\left(A_{0} \omega_{0}\right)^{2}}{N r_{F}\left(1-e^{2}\right)}
$$

On a qualitative level our model satisfactorily reproduces the observed phenomenology: a large intruder migrates to the top of a vibrated bed, and the rise velocity increases with the intruder size. The solutions (68) and (71) are plotted in Figs. 3 and 4. Our results resembles the experimental intruder height time evolution described in Refs. [5] and [6]. However the model can not describe the intermittent ascent of the intruder since we calculate the mean velocities. Using the following model parameters: mass particle density of 2.7 $\mathrm{gcm}^{-3}$ (Aluminum), $r_{F}=0.1 \mathrm{~cm}, e=0.95, \nu=0.7, N=5 \times 10^{3}, \mathrm{~g}=100$ $\mathrm{cms}^{-2}, r_{0}=L / 3$ and $\Gamma_{0}=1.33$ we obtain that the order of magnitude of $z(t)$ (Fig. 4) coincides with the values reported by Cooke et al. (See Fig. 3 Ref. $[6])$.

From the settling velocity $u_{s}(70)$ we show explicitly the dependence on size. This is proportional to the size ratio $\phi$ and the granular temperature differences $\Delta T_{g}$ which also depends on the size ratio. Its agrees with the experimental fact that the larger the radius of the intruder, the faster is the ascent, reported by Duran et al. [5]. The plotted solution (70), describes qualitatively the experimental results of Ref. [5], for $\phi>4$, show in Fig. 5. In this experiment, Duran et al., claim the experimental evidence of a segregation size threshold at $\phi_{c}=3.3$, below which the intruder does not exhibit any upwards motion. Our model's continuous aspect doesn't allow for the existence of this threshold. We argue that this discrepancy comes from the fact that experimental measures in this regime should be very difficult to carrying out. 


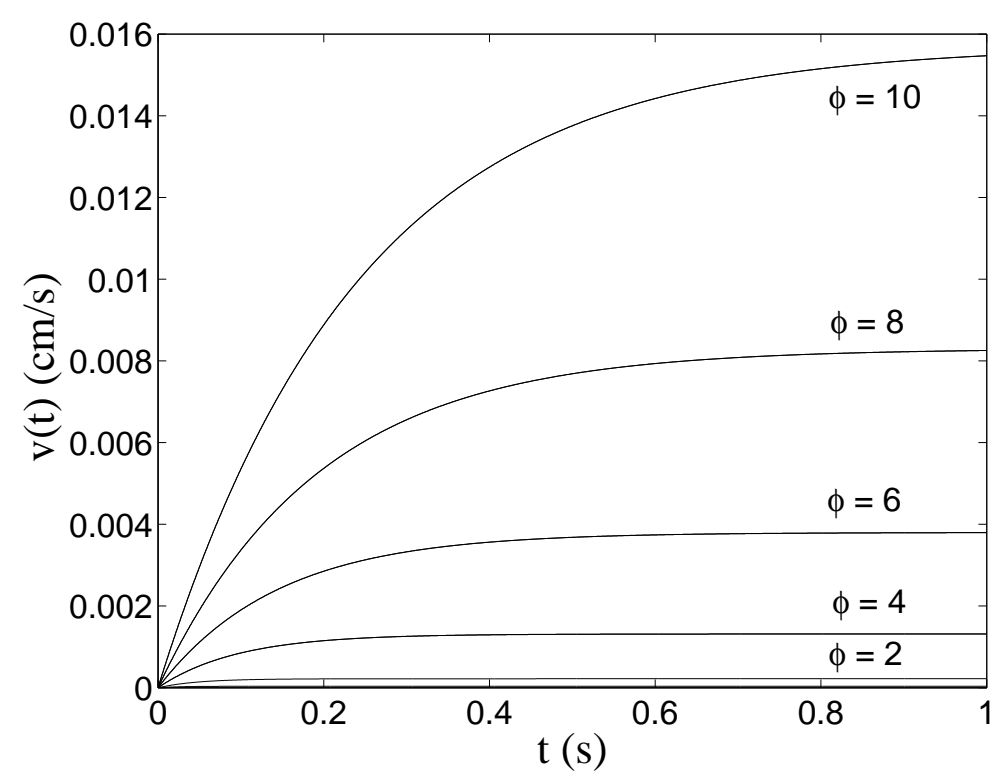

Fig. 3. Intruder segregation velocity $u(t)$. The parameters are: mass particle density of $2.7 \mathrm{gcm}^{-3}$ (Aluminum), $r_{F}=0.1 \mathrm{~cm}, e=0.95, \nu=0.7, N=5 \times 10^{3}, \mathrm{~g}=100$ $\mathrm{cms}^{-2}, r_{0}=L / 3$ and $\Gamma_{0}=1.33$.

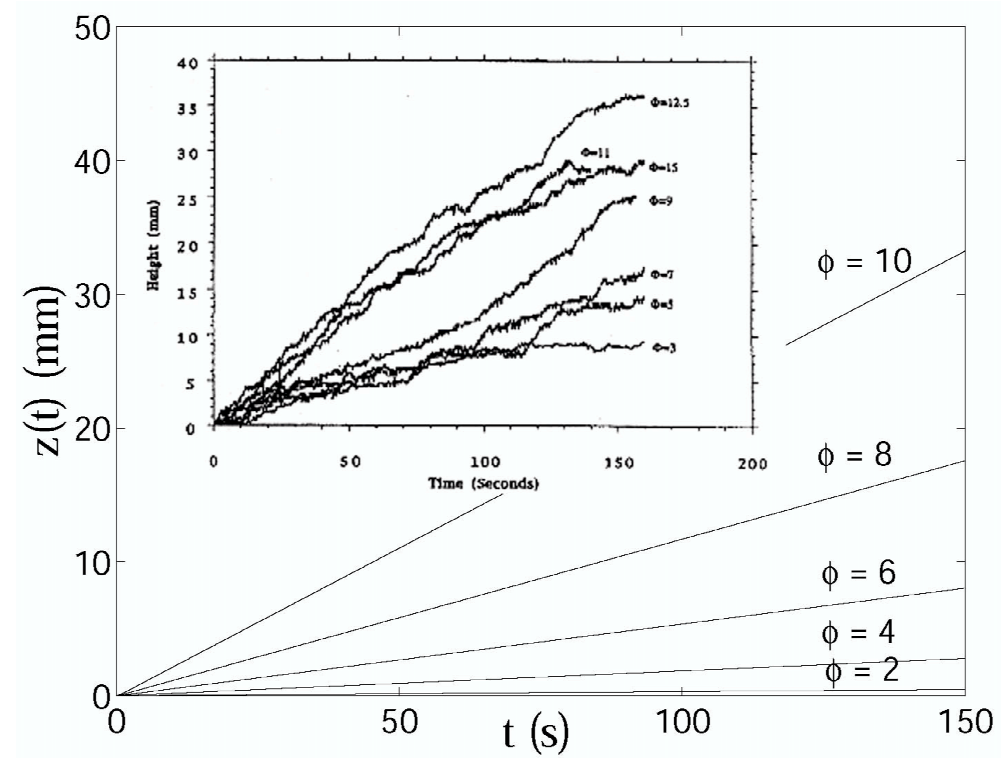

Fig. 4. Intruder height time dependence $z(t)$. The parameters are: mass particle density of $2.7 \mathrm{gcm}^{-3}$ (Aluminum), $r_{F}=0.1 \mathrm{~cm}, e=0.95, \nu=0.7, N=5 \times 10^{3}$, $\mathrm{g}=100 \mathrm{cms}^{-2}, r_{0}=L / 3$ and $\Gamma_{0}=1.33$. Inset: Measured intruder height (Fig. 3, Ref.[6]).

\section{Conclusions}

We derived a phenomenological continuum description for particle size segregation in granular media. We propose a buoyancy-driven segregation mech- 


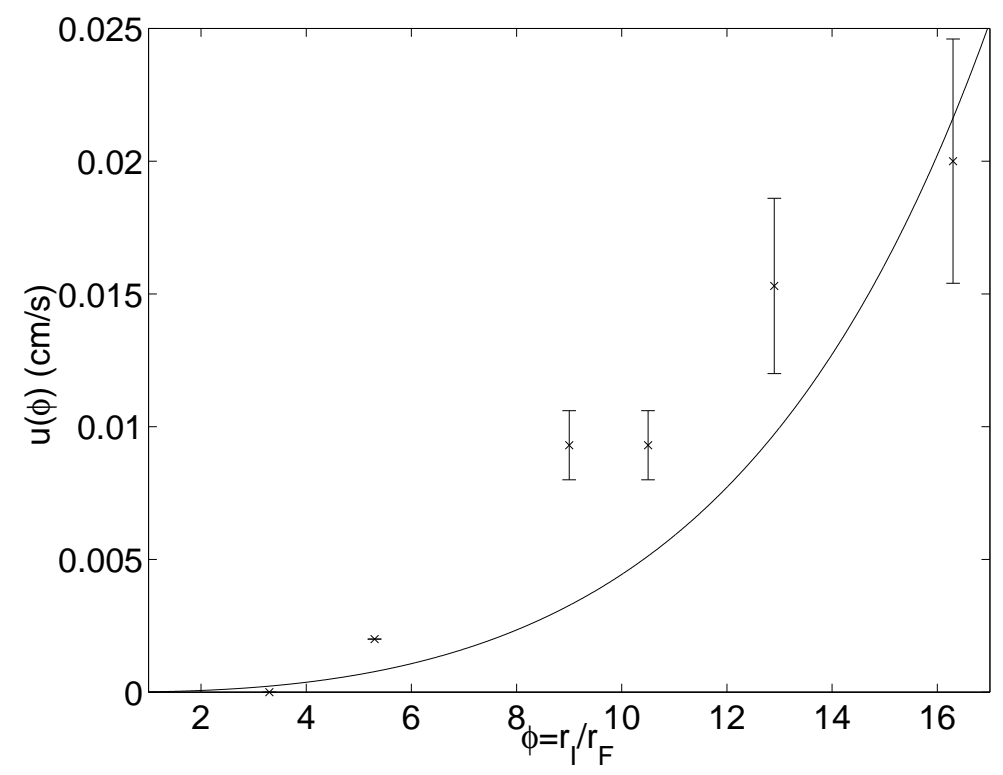

Fig. 5. Intruder segregation velocity dependence on $\phi$. The parameters are: mass particle density of $2.7 \mathrm{gcm}^{-3}$ (Aluminum), $r_{F}=0.75 \mathrm{~cm}, e=0.95, \nu=0.7$, $N=5 \times 10^{3}, \mathrm{~g}=100 \mathrm{cms}^{-2}, r_{0}=L / 3$ and $\Gamma=1.25$. The data points come from Ref.[5].

anism caused by the dissipative nature of the collisions between grains. The collisional dissipation rate naturally leads to a local temperature difference among the region around the intruder and the medium without intruder. In this model we proposed that the intruder's presence develops a temperature gradient in the system which gives origin to a difference of densities. The granular temperature difference is due to the fact that the number of collisions on the surface increases with the size of the intruder, but the local density of dissipated energy diminishes. So, the region around the intruder its hotter than the region without intruder. From this temperature difference we can conclude that we have a change in the density of the granular fluid. This lead to a buoyancy force that is the responsible for the intruder's upward movement.

In this work we made use of the tools of kinetic theory of gases to calculate the granular temperature. We observed a breakdown of the energy equipartition. And this is in agreement with other reported experiments and models. In a certain sense our theory unifies the different aspects observed in the size segregation phenomenon. Explicit solutions of the dependence of height and velocity are calculated. The geometrical effect of a segregation threshold is not supported by our model. The intruder size dependence appears naturally in our model.

It seems that in most of the segregation experiments the granular convection is unavoidable $[4-7,10,11,20]$. It is also important to note that, changes in the side walls can induce a transition to flow downward at the center of the container 
and upward along the boundaries $[62,63]$. This situation was observed in the experiments of Knight et al., [4] where the intruder particle moves downward in the middle of a conical container. However, in spite of the convective flow appeared in experimental setups, in the size-dependent regime there is not discernible convective flow in the center of the bed $[2,3,5-7,10,13,18]$, and we can conclude that convection had no influence (in this regime) on the intruder motion.

Very recently it has been shown experimentally [59] and by computer simulations [58], that the convection phenomenon in granular fluids comes from the effect of spontaneous granular temperature gradients, due to the dissipative nature of the collisions. This temperature gradient leads to a density variations. The convection rolls are caused by buoyancy effects initiated by enhanced dissipation at the walls and the tendency of the grains at the center to rise. So, this segregation mechanism could be described in the hydrodynamic framework proposed in this work subject to the appropriate boundary conditions. In the convection regime an additional drag force should appear coupling the intruder's movement with the bulk convection stream. Further investigation is required to deduce the forces associated with the convection drag on the intruder and the role of the container geometry.

In this work we only considered the case of the size-dependence on the segregation of a single intruder in a granular medium. The interplay between the intruder size and material density dependence will be the subject of future work.

\section{Acknowledgments}

We thank M. Alam for helpful comments on the manuscript. One of the authors (L.T.) would like to thank A.R. Lima for friendly support, Prof. Sidney R. Nagel for discussions concerning the experimental aspects of the segregation problem and convection, and the ICA-1 for their hospitality while part of this project was carried out.

\section{Appendix}

\section{A Transport coefficients}

In this appendix the prefactors appearing in Eqs.(9) and (64) are derived. Using a Chapman-Enskog procedure for the solution of the Enskog trans- 
port equation, the transport coefficients for nearly elastic particles have been derived in Refs. [28] and [32].

In $2 D$ the thermal conductivity $\kappa$ is $[28]$

$$
\kappa=3 n r_{F}\left(\frac{\pi}{m_{F}}\right)^{1 / 2}\left[1+\frac{1}{3} \frac{1}{G}+\frac{3}{4}\left(1+\frac{16}{9 \pi}\right) G\right] T_{g}^{1 / 2}
$$

where $G$ is $\nu g_{0}, g_{0}$ is the $2 D$ pair correlation function given in Eq.(7), and $\nu$ is the area fraction $\nu=n \pi r_{F}^{2}$. It is convenient to express Eq.(A.1) introducing the prefactor $\kappa_{0}$ defined as

$$
\kappa_{0} \equiv 3 n r_{F}\left(\frac{\pi}{m_{F}}\right)^{1 / 2}\left[1+\frac{1}{3} \frac{1}{G}+\frac{3}{4}\left(1+\frac{16}{9 \pi}\right) G\right] .
$$

The result (A.1) takes the form

$$
\kappa=\kappa_{0} \sqrt{T_{g}}
$$

In $3 D$ the thermal conductivity is [32]

$$
\kappa=\frac{15}{8} n r_{F}\left(\frac{\pi}{m_{F}}\right)^{1 / 2}\left[1+\frac{5}{24} \frac{1}{G}+\frac{6}{5}\left(1+\frac{32}{9 \pi}\right) G\right] T_{g}^{1 / 2},
$$

where $G$ is $\nu g_{0}, g_{0}$ is the $3 D$ pair correlation function given in Eq.(8), and $\nu$ is in this case the volume fraction $\nu=4 \pi n r_{F}^{3} / 3$. In $3 D$ the prefactor $\kappa_{0}$ is defined as

$$
\kappa_{0} \equiv \frac{15}{8} n r_{F}\left(\frac{\pi}{m_{F}}\right)^{1 / 2}\left[1+\frac{5}{24} \frac{1}{G}+\frac{6}{5}\left(1+\frac{32}{9 \pi}\right) G\right]
$$

The shear viscosity $\mu$ in $2 D$ is [28]

$$
\mu=\frac{1}{4} n r_{F}\left(\pi m_{F}\right)^{1 / 2}\left[2+\frac{1}{G}+\left(1+\frac{8}{\pi}\right) G\right] T_{g}^{1 / 2} .
$$

It is convenient to express Eq.(A.6) introducing the prefactor $\mu_{0}$ defined as

$$
\mu_{0}=\frac{1}{4} n r_{F}\left(\pi m_{F}\right)^{1 / 2}\left[2+\frac{1}{G}+\left(1+\frac{8}{\pi}\right) G\right] .
$$

So, the result (A.6) takes the form

$$
\mu=\mu_{0} \sqrt{T_{g}}
$$


In $3 D$ the shear viscosity is [32]

$$
\mu=\frac{1}{3} n r_{F}\left(\pi m_{F}\right)^{1 / 2}\left[1+\frac{5}{16} \frac{1}{G}+\frac{4}{5}\left(1+\frac{12}{\pi}\right) G\right] T_{g}^{1 / 2}
$$

and the prefactor $\mu_{0}$ in $3 D$ is defined as

$$
\mu_{0}=\frac{1}{3} n r_{F}\left(\pi m_{F}\right)^{1 / 2}\left[1+\frac{5}{16} \frac{1}{G}+\frac{4}{5}\left(1+\frac{12}{\pi}\right) G\right] .
$$

\section{B Thermal expansion coefficient}

We can consider the volume of the system as a function of the granular temperature and the pressure $V=V\left(T_{g}, p\right)$. A change in the granular temperature $d T_{g}$ and the pressure $d p$, leads to the corresponding change in the volume $d V$

$$
d V=\left(\frac{\partial V}{\partial T_{g}}\right)_{p} d T_{g}+\left(\frac{\partial V}{\partial p}\right)_{T_{g}} d p
$$

As we have supposed that the pressure of the system is more or less constant, we can approximate $d p \sim 0$. The increment of volume $d V$ with an increment of the granular temperature $d T_{g}$ is

$$
d V=\left(\frac{\partial V}{\partial T_{g}}\right)_{p} d T_{g}
$$

Thus,

$$
\frac{d V}{d T_{g}}=\left(\frac{\partial V}{\partial T_{g}}\right)_{p}
$$

or

$$
\left(\frac{\partial V}{\partial T_{g}}\right)_{p}=\left[\left(\frac{\partial T_{g}}{\partial V}\right)_{p}\right]^{-1}
$$

and in terms of the number density $n$, we have

$$
\left(\frac{\partial n}{\partial T_{g}}\right)_{p, N}=\left[\left(\frac{\partial T_{g}}{\partial n}\right)_{p, N}\right]^{-1} .
$$


From the definition of the coefficient of thermal expansion Eq.(57), and from the above statement, we find

$$
\alpha=-\frac{1}{n}\left(\frac{\partial n}{\partial T_{g}}\right)_{p, N}=-\frac{1}{n}\left[\left(\frac{\partial T_{g}}{\partial n}\right)_{p, N}\right]^{-1} .
$$

The partial derivative $\left(\partial T_{g} / \partial n\right)_{p, N}$ can be calculated from the equation of state (B.7). In $2 D$ the equation of state is

$$
p=n T_{g}\left[1+(1+e)\left(\frac{\nu}{1-\nu}+\frac{9}{16} \frac{\nu^{2}}{(1-\nu)^{2}}\right)\right] .
$$

where $\nu=n \pi r_{F}^{2}$. So, an elementary calculation leads to

$$
\begin{aligned}
\left(\frac{\partial T_{g}}{\partial n}\right)_{p, N}= & (\nu-1) \frac{p}{n^{2}} \\
& \times \frac{\left[\frac{7}{16}(1+e)-1\right]\left(\nu^{3}-3 \nu^{2}\right)-(1-2 e) \nu+1}{\left\{\left[\frac{7}{16}(1+e)-1\right] \nu^{2}+(1-e) \nu-1\right\}^{2}}
\end{aligned}
$$

From (B.6) one obtains:

$$
\begin{aligned}
\alpha= & \frac{n}{(1-\nu) p} \\
& \times \frac{\left\{\left[\frac{7}{16}(1+e)-1\right] \nu^{2}+(1-e) \nu-1\right\}^{2}}{\left[\frac{7}{16}(1+e)-1\right]\left(\nu^{3}-3 \nu^{2}\right)-(1-2 e) \nu+1}
\end{aligned}
$$

Using the equation of state (B.7) we can express $\alpha$ in function of the granular temperature

$$
\begin{aligned}
\alpha= & \frac{1}{T_{g}}\{(1-\nu)[1+(1+e) G]\}^{-1} \\
& \times \frac{\left\{\left[\frac{7}{16}(1+e)-1\right] \nu^{2}+(1-e) \nu-1\right\}^{2}}{\left[\frac{7}{16}(1+e)-1\right]\left(\nu^{3}-3 \nu^{2}\right)-(1-2 e) \nu+1}
\end{aligned}
$$

this is

$$
\alpha=\frac{1}{T_{g}} C(\nu)
$$

where the correction coefficient due to the density of the system is defined as 


$$
\begin{aligned}
C(\nu) \equiv\{(1-\nu)[1+(1+e) G]\}^{-1} \\
\\
\times \frac{\left\{\left[\frac{7}{16}(1+e)-1\right] \nu^{2}+(1-e) \nu-1\right\}^{2}}{\left[\frac{7}{16}(1+e)-1\right]\left(\nu^{3}-3 \nu^{2}\right)-(1-2 e) \nu+1}
\end{aligned}
$$

For three dimensions the equation of state is

$$
p=n T_{g}\left[1+(1+e)\left(\frac{\nu}{1-\nu}+\frac{9}{16} \frac{\nu^{2}}{(1-\nu)^{2}}\left[3+\frac{\nu}{2(1-\nu)}\right]\right)\right] .
$$

In a similar way we find for $3 D$ that the coefficient of thermal expansion is

$$
\begin{aligned}
\alpha= & \frac{1}{T_{g}}\left\{(1-\nu)^{2}[1+2(1+e) G]\right\}^{-1} \\
& \times \frac{\left\{\nu^{3}-(2-e) \nu^{2}+(1-2 e) \nu-1\right\}^{2}}{\nu^{4}-4 \nu 3-(5-e) \nu^{2}+4 e \nu+1}
\end{aligned}
$$

and the correction coefficient $C(\nu)$ in $3 D$ is defined as

$$
\begin{aligned}
C(\nu)= & \left\{(1-\nu)^{2}[1+2(1+e) G]\right\}^{-1} \\
& \times \frac{\left\{\nu^{3}-(2-e) \nu^{2}+(1-2 e) \nu-1\right\}^{2}}{\nu^{4}-4 \nu 3-(5-e) \nu^{2}+4 e \nu+1}
\end{aligned}
$$

where $\nu=4 n \pi r_{F}^{3} / 3$.

\section{References}

[1] Physics of Dry Granular Media, edited by H. J. Herrmann, J. -P. Holvi and S. Luding, NATO ASI, Ser. E, Vol. 350 (Kluwer Academic Publishers, Dordrecht, 1998).

[2] A. Rosato, K. J. Strandburg, F. Prinz and R. H. Swendsen, Phys. Rev. Lett. 58 (1987) 1038.

[3] J. Duran, J. Rajchenbach and E. Clément, Phys. Rev. Lett. 70 (1993) 2431.

[4] J. B. Knight, H. M. Jaeger and S. R. Nagel, Phys. Rev. Lett. 70 (1993) 3728.

[5] J. Duran, T. Mazoi, E. Clément and J. Rajchenbach, Phys. Rev. E50 (1994) 5138.

[6] W. Cooke, S. Warr, J. M. Huntley and R. C. Ball, Phys. Rev. E53 (1996) 2812. 
[7] L. Vanel, A. D. Rosato and R.N. Dave, Phys. Rev. Lett. 78 (1997) 1255.

[8] D. Brone and F. J. Muzzio, Phys. Rev. E56 (1997) 1059.

[9] T. Shinbrot and F.J. Muzzio, Phys. Rev. Lett. 81 (1998) 4365.

[10] K. Liffman, K. Muniandy, M. Rhodes, D. Gutteridge and G. Metcalfe, Granular Matter 3 (2001) 205.

[11] M. E. Möbius, B. E. Lauderdale, S. R. Nagel and H. M. Jaeger, Nature (London) 414 (2001) 270.

[12] N. Burtally, P. J. King and M. R. Swift, Science 295 (2002) 1877.

[13] A. Rosato, F. Prinz, K. J. Standburg and R. Swendsen, Powder Technology 49 (1986) 59.

[14] R. Jullien, P. Meakin and A. Pavlovitch, Phys. Rev. Lett. 69 (1992) 640.

[15] R. Jullien, P. Meakin and A. Pavlovitch, Europhys. Lett. 22 (1993) 523.

[16] T. Pöschel and H. J. Herrmann, Europhys. Lett. 29 (1995) 123.

[17] T. Ohtsuki, D. Kinoshita, Y. Takmoto and A. Hayashi, J. Phys. Soc. Jpn. 64 (1995) 430.

[18] S. Dippel and S. Luding, J. Phys. I (Paris) 5 (1995) 1527.

[19] J. A. C. Gallas, H. J. Herrmann, T. Pöschel and S.Sokołowski, J. Stat. Phys. 82 (1996) 443.

[20] Y. Lan and A. D. Rosato, Phys. Fluids 9 (1997) 3615.

[21] E. Caglioti, A. Coniglio, H. J. Herrmann, V. Loreto and M. Nicodemi, Europhys. Lett. 43 (1998) 591.

[22] D. C. Hong, P. V. Quinn and S. Luding, Phys. Rev. Lett. 86 (2001) 3423.

[23] N. Shishodia and C. R. Wassgren, Phys. Rev. Lett. 87 (2001) 084302, ibid. 88 (2002) 109901(E).

[24] G. C. Barker, A. Mehta and M. J. Grimson, Phys. Rev. Lett. 70 (1993) 2194.

[25] R. Jullien, P. Meakin and A. Pavlovitch, Phys. Rev. Lett. 70 (1993) 2195.

[26] G. C. Barker and A. Mehta, Europhys. Lett. 29 (1995) 61.

[27] R. Jullien, P. Meakin and A. Pavlovitch, Europhys. Lett. 29 (1995) 63.

[28] J. T. Jenkins and M. W. Richman, Phys. Fluids 28 (1985) 3485.

[29] S. B. Savage, J. Fluid Mech. 194 (1988) 457.

[30] J. T. Jenkins and M. W. Richman, J. Fluid Mech. 192 (1988) 313.

[31] C. S. Campbell, Annu. Rev. Fluid Mech. 22 (1990) 57. 
[32] J. T. Jenkins, in Ref. [1], pag. 353.

[33] V. Kumaran, J. Fluid. Mech. 364 (1998) 163.

[34] V. Garzó and J. W. Dufty, Phys. Rev. E59 (1999) 5895.

[35] J. T. Jenkins and F. Mancini, Phys. Fluids A1 (1989) 2050.

[36] P. Zamankhan, Phys. Rev. E52 (1995) 4877.

[37] B. Ö. Arnarson and J. T. Willits, Phys. Fluids 10 (1998) 1324.

[38] J. T. Willits and B. Ö. Arnarson, Phys. Fluids 11 (1999) 3116.

[39] S. McNamara and S. Luding, in Proceedings of the IUTAM Symposium on Segregation in Granular Flows, edited by A. Rosato (Kluwer Academic Publishers, Dordrecht, 2000).

[40] C. Henrique, G. Batrouni and D. Bideau, Phys. Rev. E63 (2001) 011304.

[41] P. K. Haff, J. Fluid Mech. 134 (1983) 401.

[42] L. Verlet and D. Levesque, Mol. Phys. 46 (1982) 969.

[43] N. F. Carnahan and K. E. Starling, J. Chem. Phys. 51 (1969) 635.

[44] S. Luding and S. Strauss, in Granular Gases, edited by T. Pöshel and S. Luding (Springer, Berlin, 2001)

[45] S. Chapman and T. G. Cowling, The Mathematical Theory of Non-uniform gases (Cambridge University Press, Cambridge, England, 1970).

[46] G. A. Mansoori, N. F. Carnahan, K. E. Starling and T. W. Leland Jr., J. Chem. Phys. 54 (1971) 1523.

[47] J. T. Jenkins and F. Mancini, J. App. Mech. 54 (1987) 27.

[48] Handbook of Mathematical Functions, edited by M. Abramowitz and I. A. Stegun (Dover, New York, 1965).

[49] R. D. Wildman and D. J. Parker, Phys. Rev. Lett. 88 (2002) 064301.

[50] K. Feitosa and N. Menon, Phys. Rev. Lett. 88 (2002) 198301.

[51] I. Ippolito, et al., Phys. Rev. E52 (1995) 2072.

[52] L. Huilin, D. Gidaspow and E. Manger, Phys. Rev. E64 (2001) 061301.

[53] H.J. Herrmann, J. Phys. II (France) 3 (1993) 427.

[54] S. F. Edwards in Granular Matter: An Interdisciplinary Approach, edited by A. Mehta (Springer-Verlag, New York, 1994).

[55] H. Hayakawa and D.C. Hong, Phys. Rev. Lett. 78 (1997) 2764. 
[56] A. Barrat, J. Kurchan, V. Loreto and M. Sellitto, Phys. Rev. Lett. 85 (2000) 5034; Phys. Rev. E63 (2001) 051301; H.A. Makse and J. Kurchan, Nature (London) 415 (2002) 614.

[57] P. Sunthar and V. Kumaran, Phys. Rev. E60 (1999) 1951.

[58] R. Ramirez, D. Riso and P. Cordero, Phys. Rev. Lett. 85 (2000) 1230.

[59] R. D. Wildman, J. M. Huntley and D. J. Parker, Phys. Rev. Lett. 86 (2001) 3304 .

[60] O. Zik, J. Stavans and Y. Rabin, Europhys. Lett. 17 (1992) 315.

[61] R. Albert, M. A. Pfeifer, A.-L. Barabási and P. Schiffer, Phys. Rev. Lett. 82 (1999) 205.

[62] J. B. Knight, Phys. Rev. E55 (1997) 6016.

[63] E. L. Grossman, Phys. Rev. E56 (1997) 3290. 